\title{
Les ouvriers au cinéma
}

Workmen through cinema

Matéo Alaluf

\section{OpenEdition}

\section{Journals}

Édition électronique

URL : https://journals.openedition.org/itti/1243

DOI : 10.4000/itti.1243

\section{Éditeur}

Université de Poitiers

\section{Référence électronique}

Matéo Alaluf, "Les ouvriers au cinéma », Images du travail, travail des images [En ligne], 2 | 2016, mis en ligne le 01 septembre 2016, consulté le 25 juillet 2021. URL : http://journals.openedition.org/itti/1243 ; DOI : https://doi.org/10.4000/itti. 1243

Ce document a été généré automatiquement le 25 juillet 2021.

Images du travail, travail des images 


\title{
Les ouvriers au cinéma
}

\author{
Workmen through cinema
}

\author{
Matéo Alaluf
}

1 Les procédures scientifiques se prétendent "objectives" dans la mesure où elles refusent de laisser la subjectivité du chercheur interférer avec les résultats de la recherche. La photographie et le cinéma apparaitront d'abord aux chercheurs comme une possibilité qui leur serait offerte pour reproduire le réel, sans le modifier. À une époque où le positivisme caractérisait les sciences sociales, la capacité attribuée à des machines de traduire la réalité en échappant aux biais des habitudes perceptives des chercheurs pouvait, au début du siècle passé, conforter la prétention de faire science non seulement avec les phénomènes naturels mais également sociaux. S'agissant des activités de travail, la photographie a tenté de saisir les opérations en décomposant les gestes de l'opérateur (Gilbreth, Waxweiler, Marey...), le cinéma l'a tenté par la mise en mouvement.

\section{La mise en intrigue du travail}

2 En principe objectives, les procédures scientifiques n'en relèvent pas moins de finalités qui n'ont pas pour origine la réalité observée mais bien des raisons propres à l'observateur. Les gestes pour effectuer une opération, mis en scène par le cinéma, ne suffisent pas à rendre compte de la situation de travail. La tâche effectuée ne permet pas de qualifier l'ouvrier ou l'ouvrière. La situation de travail résulte tout autant de conditions hors travail. La représentation filmée de la seule opération isolée de la vie salariale se rapporte à l'opérateur abstrait et sert alors à prescrire des normes à chaque opération.

3 Tout comme l'archive seule ne rend pas compte de la réalité, l'exposition à l'objectif ne la révèle pas davantage. Le premier film de l'histoire du cinéma, La sortie de l'usine Lumière à Lyon (1895), est aussi le premier film sur le travail. 
5

Trois versions sont connues. Seule dans la première, les ouvrières ont leurs habits de travail. Dans les autres versions elles portent leurs vêtements du dimanche. Le film rend compte de la performance technique : appareil de projection, film $35 \mathrm{~mm}$ (double jeu de perforations longues à la différence du Kinétographe de Thomas Edison) et cinématographe (marque déposée). On parle aussi de vue photographique animée.

Il montre les ouvrières (femmes) et les employés (hommes) qui quittent l'usine. Le film est instructif en ce qui concerne le travail plus par ce qu'il ne montre pas que par ce qu'il montre. Il ne nous apprend rien des conditions de travail aux usines Lumière que nous savons par ailleurs être dures et où les "fortes têtes " étaient licenciées. En montrant la sortie de l'usine, le film exclut du regard le travail à l'atelier tout comme la vie d'après le travail. Il rend compte de la performance de la caméra mais ne nous instruit pas d'avantage sur les ouvrières et les ouvriers qui quittent l'usine. La caméra trace ainsi la ligne de partage entre le visible et l'invisible.

7 Le cinéma ne s'est pas contenté de filmer la sortie d'une usine. Il s'est rapidement trouvé au service par exemple des organisateurs du travail ou des chercheurs, comme des cinéastes. La scénarisation permettra alors de rendre compte du travail et de l'entreprise. A visit To Peek Frean \& Co's Bisquit works (Bermondsey, London), réalisé par les studios Cricks \& Sharp en 1906 présenté comme un des premiers documentaires sur le travail, reconstitue toute la filière de production de biscuits (extraction de charbon nécessaire aux machines, réception des matières, fabrication, pause de midi, conditionnement et commercialisation des biscuits). Ainsi, le lieu de production, l'atelier, inséré dans la société devient un lieu central. Le film met en scène toutes les phases de travail conduisant à la fabrication et à la distribution des biscuits. La pause de midi permet de sortir de l'usine et rend compte de l'importance numérique des ouvriers qui quittent leurs entreprises respectives et se répandent en ville. L'atelier pénètre ainsi la ville et imprime son rythme à la vie. Le travail de chaque ouvrier de la biscuiterie constitue un moment d'un flux de production et ceux qui travaillent appartiennent au groupe ouvrier. "La mise en intrigue » du travail a donc permis très tôt de représenter au cinéma l'ouvrier qui ne se réduit ni aux seules personnes quittant l'usine ni aux seuls actes d'un opérateur sur sa machine.

Ce média ne peut être affiché ici. Veuillez vous reporter à l'édition en ligne http:// journals.openedition.org/itti/1243

\section{Le cinéma du réel}

9 L'homme à la caméra de Dziga Vertov (1929, 95min) filme le travail du cameraman qui filme le travail. Il dévoile (rend visible) les activités multiples qui font la journée d'une ville en explorant toutes les facettes qui font le quotidien des habitants. Le prologue expose l'appareillage cinématographique (caméra, film, montage, appareil et salle de projection). La journée est découpée en 6 chapitres : La ville dort; la ville s'éveille; la ville en activité ; la ville travaille ; les sports ; les loisirs. En même temps, dans chaque chapitre trois temporalités se superposent: la ville comme elle est sur l'écran de 
cinéma; la ville comme elle est sur la pellicule; la ville comme elle est tout court (montage). Le cinéma doit montrer selon Vertov la vie et non la fiction. L'homme à la caméra tente de montrer le monde tel qu'il est aux travailleurs. Le travail des autres travailleurs comme celui de leurs exploiteurs. Il vise à démêler l'illusion de la réalité. Ce que l'on voit n'est que représentation. Le cinéma du réel ambitionne de casser la représentation rassurante $\mathrm{du}$ monde que véhicule la fiction et l'industrie cinématographique. Alors cependant qu'il refuse la fiction, le cinéma du réel n'est à son tour aussi que représentation. Le travail apparaît comme la condition d'existence de la ville filmée par celui qui travaille à la caméra. On voit d'abord des machines à l'arrêt et des hommes et des femmes endormis ou au ralenti, ensuite ils sont en mouvement et enfin ils sont réunis dans le travail (hommes machines). L'homme à la caméra s'incorpore également au travail (c'est un travailleur comme un autre). Il participe, comme les autres travailleurs à l'élaboration et au fonctionnement de la société. La capacité du cinéma à reproduire la réalité lui permet de nourrir une double ambition : celle, en décloisonnant les activités, de rendre la société transparente à elle même et celle de participer à l'émancipation ouvrière.

Ce « film dans le film » est à l'origine de ce que Vertov désigne comme « ciné-œil » (œil mécanique) et que l'on appellera par la suite cinéma du réel, cinéma direct ou cinéma vérité. On suit le cameraman qui filme, le montage d'une séquence et le public assistant à la projection est le sujet (et non plus le spectateur passif) de ce qui est filmé. Ce cinéma sans scénario se veut un regard spécifique et distinct de la littérature, de la peinture, du théâtre comme de la science, sur le monde. L'homme à la caméra a profondément marqué toute l'histoire du cinéma.

Illustration 1. Affiche originale du film L'homme à la caméra

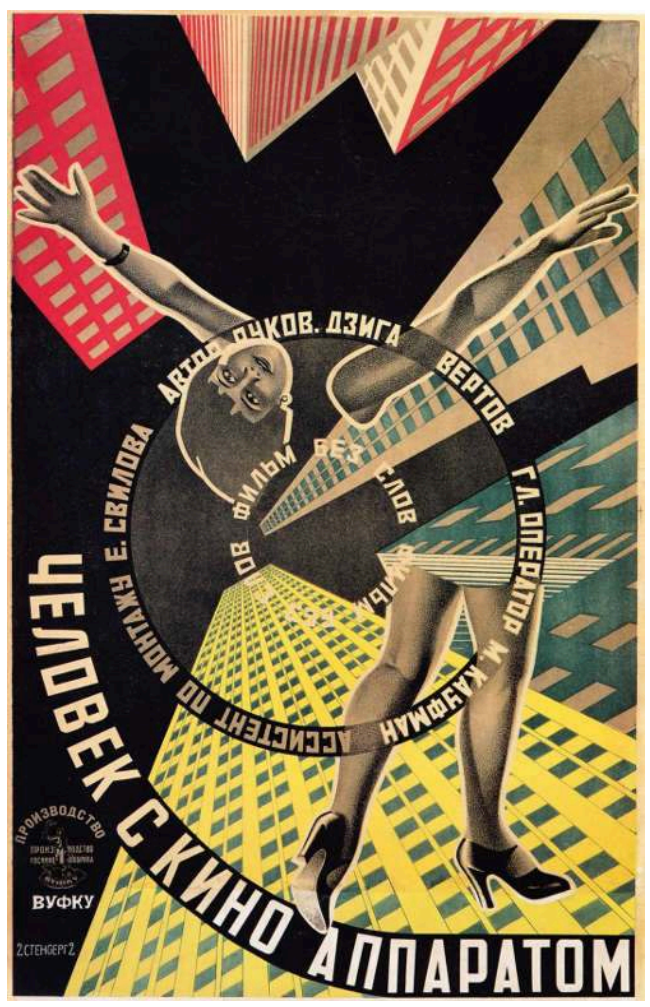


11 Misère au borinage (1933) de Henri Storck et Joris Ivens est un classique du cinéma du réel. En mêlant fiction et réalité, le documentaire explore l'exploitation ouvrière et les conditions de vie des ouvriers mineurs. Réalisé par un ostendais (Storck) et un hollandais (Ivens), Misère au borinage est considéré comme l'acte fondateur du cinéma réaliste wallon. Déjà s'envole la fleur maigre (1960) de Paul Meyer qui aborde la question des ouvriers étrangers au borinage, en constitue la suite. Les enfants du borinage (1999) de Patrick Jean revisitera beaucoup plus tard la même misère sociale sous forme d'une lettre à Henri Storck. Ces trois films témoignent de la misère des bassins miniers mais aussi de la puissance politique des mineurs dans les luttes sociales. Les frères Dardenne s'inscrivent aujourd'hui dans cette même tradition.

Pour les besoins de leur film, Storck et Ivens avaient simulé une manifestation de mineurs avec des figurants défilant derrière un portrait de Marx. La gendarmerie prenant cette scène de cinéma pour une manifestation réelle chargea les figurants qui n'en étaient pas moins des ouvriers mineurs, permettant ainsi à la camera de filmer une vraie charge de gendarmerie sur des manifestants. Walter Benjamin écrit, à propos de Misère au borinage, que n'importe quel passant a sa chance de devenir figurant dans un film et de figurer ainsi dans une œuvre d'Art. Siegfried Kracauer, en dialogue avec Walter Benjamin, a forgé l'expression de "camera-reality" pour désigner une relation entre la caméra et le réel affranchi de toute problématique de représentation. La "reproduction » de la société par l'objectif, en révélant des aspects non perçus ou involontaires, aboutit ainsi à une autre perception de la réalité1.

La "caméra-réalité » rend compte d'une relation au monde lorsque l'observateur est appareillé d'une caméra. Ce regard est à la fois réaliste (reproduction de la réalité) et formateur (tenant de l'art). Il positionne le film par rapport à la réalité matérielle. De ce point de vue, selon Kracauer, fiction et documentaire ne fonctionnent pas différemment. Adaptant au cinéma la théorie de Benjamin sur la reproductibilité technique de l'œuvre d'art, Kracauer considère que le film doit procéder, à la différence de l'art traditionnel qui impose une forme sur une matière, en faisant sienne les qualités de la caméra en tant que technique de reproduction.

Le "cinéma direct», courant qui se développe à partir de la fin des années 1950, principalement aux États-Unis et au Canada, se pose le problème du réel dans les mêmes termes. Le film, en raison de son objectivité mécanique, est considéré comme instrument de vérité. Il se propose de capter directement le réel et d'agir sur la réalité. Les squatters (1958) de Michel Brault au Québec et Don Alan Pennebaker, Richard Leacock ou Frederick Wiseman aux États-Unis sont parmi les principaux représentants de ce courant. Chronique d'un été (1961) de Jean Rouch et Edgar Morin participe à ce courant. Angelo est ouvrier à Renault-Billancourt. Il se plaint de la routine qui envahit sa vie. Il est le pendant ouvrier des autres personnages du film tout en mettant à mal la figure du prolétaire, ouvrier conscient et organisé, porteur d'un projet socialiste. Son travail est monotone, parcellaire et dépourvu d'intérêt et sa vie se résume à " $9 \mathrm{~h}$ de travail, dormir, aller travailler». Angelo voudrait fuir l'usine par la promotion professionnelle en suivant des cours ou en allant faire les marchés.

Ce média ne peut être affiché ici. Veuillez vous reporter à l'édition en ligne http:// 

sortie de l'usine lumière à Lyon, l'attitude consistant à filmer la vie telle qu'elle est accompagne le cinéma. La représentation du monde du travail dans « le cinéma direct " fera «surgir des individualités ouvrières » dans leur inscription dans la société. Les auteurs évoquent notamment, outre Chronique d'un été, Les enfants du néant de Michel Brault et Annie Tresgot (1968), filmé dans les usines Citroën de Rennes et Humain trop humain de Louis Malle (1972), tourné sur le même site, sans aucune voix off qui présente «l'engagement des corps au travail » de manière à dégager de la chaîne de montage « un sentiment physique de fatigue et d'ennui. » ( Hatzfeld, Rot, Michel, 2009, 67)

Capter le réel par le cinéma et le rendre visible dans l'espace public est bien un projet autant politique qu'esthétique assumé par ce cinéma.

\section{Les temps modernes} survivre dans un monde industrialisé. Chronique de la " grande dépression ", satire du travail à la chaîne, réquisitoire contre le chômage ce film est encore bien plus que cela. Le travail industriel apparaît en fait comme la métaphore des formes de rationalisation des sociétés contemporaines et Charlot celui du «paria $»^{2}$ qui lui résiste. C'est précisément parce qu'il ne se laisse pas réduire à la critique du taylorisme et de la société industrielle que Les temps modernes est un des plus grands films de l'histoire du cinéma.

19 Charlot n'est pas seul dans cette bataille avec le monde moderne. Revenant en Amérique, Chaplin avait connu l'actrice Paulette Goddard, qui devait rester pendant plusieurs années une compagne idéale dans sa vie privée. Elle a inspiré le personnage de la Gamine dans Les Temps modernes : une jeune fille dont le père a été tué pendant une grève, et qui s'allie à Charlot. Ils se rencontrent dans un panier à salade où la fille a été jetée après avoir volé un pain, puis ils décident de vivre ensemble, en toute innocence. Ce ne sont ni des rebelles ni des victimes, mais, écrivait Chaplin, « les deux seuls esprits vivants dans un monde d'automates. Nous sommes des enfants sans aucun sens des responsabilités. Le reste de l'humanité est accablé par ses devoirs. Nous sommes libres en esprit ».

20 C'est pourquoi, « dépasser l'image du Charlot des temps modernes », selon l'expression d'Alexandra Bidet, ne consiste pas seulement à réfuter les représentations mécaniques du travail industriel et du travail parcellaire dont la critique a d'ailleurs été tellement répétée, mais surtout à occulter la "question sociale " liée au travail ouvrier. En d'autres termes, la vie ouvrière ne se limite pas au travail et le travail ne se réduit pas à l'activité du travailleur.

\section{Le Mai ouvrier}

21 La révolte étudiante de 1968 avait occupé quasi tout l'espace médiatique du moins en ce qui concerne la presse, la radio et la télévision. Un nouveau cinéma militant se mobilisera autour du Mai ouvrier. Il restitue en priorité le Mai ouvrier minimisé dans 
l'espace médiatique par le mouvement étudiant. Il prend la suite du " cinéma direct " avec les conflits sociaux en plus.

Des cinéastes militants avaient édicté « la règle de ne pas filmer " pour ne pas capturer le réel en spectacle et ne pas se laisser capturer à son tour par le spectacle du réel mis en scène par la classe dominante. D'autres cependant filmeront dans les rues, les ateliers, les écoles et voudront enregistrer la parole subjective de tout un chacun de manière à restituer « l'effervescence des émotions politiques » et mettre en scène une « autoreprésentation du peuple ${ }^{3}$.»

En fait la notion de " cinéma militant » apparaît avec 2 films réalisés par Chris Marker en 1967 : Loin de Vietnam, avec la participation entre autres de Joris Ivens, Jean-Luc Godart et Claude Lelouch, sur l'opposition à la guerre du Vietnam et $A$ bientôt, j'espère, sur la grève de la Rhodiaceta. Chris Marker sera aussi à l'origine de la création de SloneIskra, collectif de production et de diffusion qui contribuera très largement à la promotion des films militants.

Des jeunes étudiants en cinéma, Jacques Willemont, Pierre Bonneau, Liane EstiezWillemont et Roland Chicheportiche se pésentent en juin 1968 devant l'usine Wonder pour filmer son occupation. Ils tournent un plan séquence de 9 minutes, La reprise $d u$ travail aux usines Wonder, qui deviendra le film emblématique de 1968.

Ce média ne peut être affiché ici. Veuillez vous reporter à l'édition en ligne http:// journals.openedition.org/itti/1243

L'objectif dominant du cinéma militant était de filmer les ouvriers et les grèves occultés par les médias. Ainsi par exemple la grève, l'occupation et l'autoproduction de l'usine Lip à Besançon de 1973 à 1976 fera l'objet des films de Carole Roussopoulos (Lip. Monique, 1973), de Chris Marker (La grève des travailleurs de Lip, 1974), de Dominique Dubosc (Le goût du collectif, 1977) et de Richard Copans (À pas lentes, 1976). Plus récemment, Christian Rouaud a revisité Lip avec Lip. L'imagination au pouvoir (2007).

Le groupe Medvedkine qui s'est constitué autour de Chris Marker en 1967 a marqué incontestablement ce cinéma. Le film de Bruno Muel, Classe de lutte (1969) se situe dans le prolongement de À bientôt j'espère. Il tourna ensuite Week-end à Sochaux» (1970) et Avec le sang des autres (1974). Embauché comme OS en 1968 chez Peugeot comme opérateur et agent de fabrication, affilié à la CGT, Christian Corouge joue un rôle important. Il se liera avec Michel Pialoux, sociologue de la condition ouvrière. Ils cosigneront ensuite des articles dans Actes de la recherche en sciences sociales, revue dirigée par Pierre Bourdieut. Alors que le cinéma militant voulait en finir avec le regard de l'ethnologue et du sociologue de manière à mettre un terme à l'appropriation indue par les «parisiens" du vécu ouvrier, ce cinéma sera un lieu de rencontre fécond entre cinéastes, ouvriers et intellectuels praticiens des sciences sociales.

Les ouvriers de l'après mai seront aussi très présents dans les films de fiction. Citons simplement Élise ou la vraie vie de Michel Drach (1969) ou en Italie, un film emblématique de cette période, La classe ouvrière va au paradis d'Elio Petri (1971). La représentation cinématographique des ouvriers sera aussi celle des ouvrières, souvent au premier plan. Les OS et les travailleurs immigrés apparaissent également de manière centrale. La dénonciation ne visera pas seulement le patronat et l'État mais s'adressera aussi aux organisations et appareils politiques et syndicaux. Ce cinéma est, dans les termes de Jean-Louis Comolli, celui « des émotions politiques». 


\section{L'ouvrier d'après la classe}

\section{financiarisation et la mondialisation des activités se traduit aussi par l'apparition de} thèmes nouveaux. La critique des nouvelles formes de management et d'évaluation du travail se retrouvent dans de nombreuses productions. On peut citer parmi d'autres: Ressources humaines (1999) de Laurent Cantet, Violences des échanges en milieu tempéré (2003) de Jean-Marc Moutout et Rien de personnel (2009) de Mathias Gökalp. La perte d'emploi, le chômage et la recherche d'emploi sont largement abordés et traduisent en creux l'importance croissante du travail lorsque l'emploi est rationné. L'emploi du temps (2001) de Laurent Cantet et La gueule de l'emploi (2011) de Didier Cros se situent dans cette catégorie. La résistance à la précarisation de l'emploi est traitée entre autres à travers une grève dans la restauration rapide dans On n'est pas des steak hachés par Alima Rouali et Anne Galland (2002). Les restructurations, privatisations et les délocalisations d'entreprises forment la trame de nombreuses productions comme Tant que chanteront les constructeurs de navires (1999) filmé sur les chantiers navals de Boel à Tamise en Flandre par Jan Vromman, et Cheminots (2010) de Luc Joulé et Sébastien Jousse. annonçait sur le plan des sciences sociales le regain d'intérêt pour le monde ouvrier. Il servira de base documentaire à Patrick Jean pour Ouvrier c'est pas la classe (2002). Celuici renoue ainsi le fil tracé naguère par Bruno Muel, Christian Corouge, Michel Pialoux et le groupe Medvedkine de Sochaux. Laurence Jourdan avec Sochaux, cadences en chaîne (2010) revient, plus tard encore à Peugeot. Sophie Bruneau et Marc-Antoine Roudil avec Ils ne mouraient pas tous mais tous étaient frappés (2008) se situent dans le prolongement des travaux de Christophe Dejours sur la souffrance au travail.

Après le cinéma militant des années 1970 et 1980, les ouvriers et leur classe s'étaient absentés de l'espace publique, supplantés par la promotion de l'individu libéré par le marché des entraves du collectif. Le retour des ouvriers se fera plus d'une dizaine 
d'années plus tard par le cinéma. Ce cinéma se distingue cependant de celui de la période précédente. Le discours politique tenu par les personnages, tout comme la voix off ont disparu ou sont réduits à leur plus simple expression. Le travail entrave la liberté et asservit le salarié, mais revêt une place centrale dans la vie des individus. La privation d'emploi comme les situations de recrutement, d'accompagnement et d'évaluation du travail, aussi humiliantes et infantilisantes soient-elles, soulignent en creux la centralité du travail. La "souffrance au travail» désormais très présente constitue l'autre face de la privation d'emploi. Lorsque la caméra explore la condition et les modes de vie ouvriers, la référence à la classe ouvrière parait moins prégnante. En contrepoint de l'injonction à l'emploi qui caractérise les politiques publiques, le cinéma permet de percevoir, comme dans L'emploi du temps de Laurent Cantet, les perspectives d'autres modes d'existence possibles.

\section{Faire politique}

Le cinéma ne fait pas nécessairement de politique lorsqu'il montre les ouvriers. Les films dont les personnages sont des ouvriers, comme les autres, racontent des histoires, ont des objectifs artistiques, éducatifs, pédagogiques, de recherche, ou encore font la promotion d'une entreprise. Dans tous les cas cependant ils contribuent à sortir le travailleur de l'ombre. Lorsque celui-ci n'est plus seulement un opérateur mais un ouvrier, il devient aussi un acteur situé dans la société et identifié à une classe.

"L'œil machinique » de la caméra, explique Jacques Rancière, "obéit à la double maîtrise de l'opérateur et celle de son sujet.» (Rancière, 2012) L'égalité de tous devant la caméra permet de mieux distinguer " l'inégalité des petits au passage des grands » et permet de percevoir le destin commun aux uns et aux autres. Le cinéma rend surtout perceptible à sa façon le fait que ceux qui ont subi une vie décidée par d'autres peuvent aussi retrouver une capacité à faire l'histoire.

Le cinéma révèle l'atelier au public. Il permet aussi aux ouvriers de quitter le périmètre privé de l'usine et leur procure une présence dans l'espace public. Dans la mesure même où la politique ordonne les conditions de vie en société, l'atelier devient générateur de politique et l'ouvrier un acteur. D'autant plus que le cinéma n'enregistre pas seulement l'événement mais crée cet événement. Il peut ainsi « rendre historique, écrit Rancière, n'importe quelle apparition derrière une fenêtre ».

En rendant l'ouvrier visible, le cinéma est engagé dans la dimension politique du travail. En révélant l'aliénation de l'ouvrier, il bouscule les conformismes de perception créés par les images. Tout compte fait on ne filme pas, tout comme on ne peint pas et on ne sculpte pas, impunément le travail.

BIBLIOGRAPHIE 


\section{Ouvrages}

Beaud S. et Pialoux M. (1999), Retour sur la condition ouvrière, Paris, Fayard.

Comolli J-L. (2009), « Mai 68 au miroir du cinéma », Manière de Voir. Le Monde Diplomatique, $\mathrm{n}^{\circ}$ 106, p. 87.

Corouge C. et Pialoux M. (1984), «Chronique Peugeot », Actes de la recherche en sciences sociales, $\mathrm{n}^{\circ}$ 52 , p. 88-95 et $n^{\circ} 54$, p. 55-69; 1985, n 57, p. 108-128 et $n^{\circ} 60$, p. $72-74$.

Gorz G. (1980), Adieux au prolétariat, Paris, Galilée.

Hatzfeld N., Rot G. et Michel A. (2009), « L'ouvrier en personne, une irruption dans le cinéma documentaire (1961-1974)», Le mouvement social, n²26, janvier-mars, p. 67-78.

Kracauer S. (2010 [1960]), Théorie du film. La rédemption de la réalité matérielle, Paris, Flammarion.

Rancière J. (2012), Figures de l'histoire, Paris, PUF.

Touraine A. (1980), L'après socialisme, Paris, Grasset.

\section{Filmographie}

Bonneau P., Estiez-Willemont L., Chicheportiche R. et Willemont J. (1968), La reprise du travail aux usines Wonder.

Brault M. (1958), Les squatters.

Brault M. et Tresgot A. (1968), Les enfants du néant.

Bruneau S. et Roudil M-A. (2008), Ils ne mouraient pas tous mais tous étaient frappés.

Cantet L. (1999), Ressources humaines.

Cantet L. (2001), L'emploi du temps.

Chaplin C. (1936), Les temps modernes.

Copans R. (1976), À pas lentes.

Frères Dardenne. (1996), La promesse.

Frères Dardenne. (1999), Rosetta.

Frères Dardenne. (2002), Le fils.

Frères Dardenne. (2005), L'enfant.

Frères Dardenne. (2014), Deux jours, une nuit.

Drach M. (1969), Élise ou la vraie vie.

Dubosc D. (1977), Le goût du collectif.

Galland A. et Rouali A. (2002), On n'est pas des steak hachés.

Gökalp M. (2009), Rien de personnel.

Guédiguian R. (1997), Marius et Jeannette.

Ivens J. et Storck H. (1923), Misère au borinage.

Jean P. (1999), Les enfants du borinage. 
Jean P. (2002), Ouvrier c'est pas la classe.

Joulé L. et Jousse S. (2010), Cheminots.

Jourdan L. (2010), Sochaux, cadences en chaîne.

Loach K. (1990), Riff Raff.

Loach K. (1993), Raining Stones.

Loach K. (1994), Lady Bird.

Loach K. (1997), Les dockers de Liverpool.

Loach K. (2000), Bread and Roses.

Loach K. (2001), The Navigators.

Lumière L. (1895), La sortie de l'usine Lumière à Lyon.

Malle L. (1972), Humain trop humain.

Marker C. (1974), La grève des travailleurs de Lip.

Moutout M. (2003), Violences des échanges en milieu tempéré.

Muel B. (1969), Classe de lutte.

Muel B. (1970), Week-end à Sochaux.

Muel B. (1974), Avec le sang des autres.

Morin E. et Rouch J. (1961), Chronique d'un été.

Petri E. (1971), La classe ouvrière va au paradis.

Rouaud C. (2007), Lip. L'imagination au pouvoir.

Roussopoulos C. (1973), Lip. Monique.

Studios Cricks et Sharp (1906), A visit To Peek Frean \& Co's Bisquit works.

Vertov D. (1929), L’homme à la caméra.

Vromman J. (1999), Tant que chanteront les constructeurs de navires.

\section{NOTES}

1. Walter Benjamin, 1936, «L'oeuvre d'art à l'époque de sa reproduction mécanique », repris in Ecrits Français, présentés par Jean-Maurice Monnoyer, Gallimard, Paris, 1991, pp. 140-171. Et Siegfried Kracauer, 2010 [1960], Théorie du film. La rédemption de la réalité matérielle, Flammarion, Paris.

2. «Paria» dans le sens que lui donne Enzo Traverso, non seulement comme «exclu» ou « victime » mais aussi résistant.

3. Comolli J-L, « Mai 68 au miroir du cinéma », Manière de Voir. Le Monde Diplomatique, $\mathrm{n}^{\circ} 106$, aoûtseptembre 2009, p. 87.

4. Corouge C. et Pialoux M. «Chronique Peugeot », Actes de la recherche en sciences sociales, (1984), $\mathrm{n}^{\circ} 52$, p. 88-95 et $\mathrm{n}^{\circ} 54$, p. 55-69; (1985), $\mathrm{n}^{\circ} 57$, p. 108-128 et $\mathrm{n}^{\circ} 60$, p. $72-74$. 


\section{RÉSUMÉS}

Le premier film de l'histoire du cinéma, La sortie de l'usine Lumière (1895), est aussi le premier film sur le travail. Depuis lors, que ce soit par la fiction, le documentaire ou le cinéma direct, le travail, les ouvriers et leur classe ont rythmé toute l'histoire du cinéma. A la différence de la représentation traditionnelle du travail par la figure de l'ouvrier qualifié, masculin de la grande industrie, le cinéma a mis en scène les ouvrières et des activités de travail diversifiées. Le projet de « reproduire » par l'image le réel a pris corps par la représentation des ouvriers au cinéma. Il ne s'agit pas tant, par cette tentative, d'illustrer le travail par des images, ni de penser le travail à partir des images, mais d'essayer de le penser avec des images de manière à faire émerger des images et des pensées nouvelles. Ce cinéma pourrait alors laisser entrevoir les possibilités d'un langage spécifique susceptible d'élargir notre intelligence du monde.

The first film in the history (of cinema), La sortie de l'usine Lumière (1895), is also the first film about work. Since then, through fiction, documentary or direct cinema, work, the workmen and their class have given rhythm to all the history of cinema. Unlike the traditional representation of work by the skilled, male, worker of the big industry, cinema put diversified workers and activities on stage. The project to "reproduce" by the image the real world was really shaped by the representation of the workmen in cinema. The question was not, by this attempt, to illustrate work by images, nor to think work starting from the images, but was an attempt to think it with images to create new images and thoughts. Then this cinema could open the possibility of a specific language able to widen our intelligence of the world.

\section{INDEX}

Mots-clés : situation de travail, cinéma direct, question sociale, cinéma militant, classe ouvrière, ouvrier sans classe

Keywords : situation of work, direct cinema, social question, engaged cinema, working class, workman without class

\section{AUTEUR}

\section{MATÉO ALALUF}

Sociologue, professeur émérite de l'Université Libre de Bruxelles (ULB) ; Il est l'auteur de nombreux ouvrages et articles qui traitent de l'emploi, de la formation, de l'histoire et de la sociologie du mouvement ouvrier et de l'immigration. Il a publié récemment : Dictionnaire du prêt-à-penser. La pensée molle, Éditions Couleur livres, Bruxelles 2014 et L'allocation universelle. Nouveau label de précarité, Éditions Couleur livres, Bruxelles 2014. 